\title{
Crk adaptor protein-induced phosphorylation of Gab1 on tyrosine 307 via Src is important for organization of focal adhesions and enhanced cell migration
}

Takuya Watanabe ${ }^{1,2}$, Masumi Tsuda ${ }^{3}$, Yoshinori Makino ${ }^{1,6}$, Tassos Konstantinou ${ }^{4}$, Hiroshi Nishihara ${ }^{1}$, Tokifumi Majima ${ }^{5}$, Akio Minami ${ }^{2}$, Stephan M Feller ${ }^{4}$, Shinya Tanaka ${ }^{1}$

${ }^{I}$ Laboratory of Molecular and Cellular Pathology, Hokkaido University Graduate School of Medicine, N15, W7, Kita-ku, Sapporo 060-8638, Japan; '2Department of Orthopaedic Surgery, Hokkaido University Graduate School of Medicine, N15, W7, Kita-ku, Sapporo 060-8638, Japan; ${ }^{3}$ Department of Pathophysiology and Signal Transduction, Hokkaido University Graduate School of Medicine, N15, W7, Kita-ku, Sapporo 060-8638, Japan; ${ }^{4}$ Cell Signalling Group, Weatherall Institute of Molecular Medicine, Oxford University, Oxford, UK; ${ }^{5}$ Department of Joint Replacement and Tissue Engineering, Hokkaido University Graduate School of Medicine, N15, W7, Kita-ku, Sapporo 060-8638, Japan

Upon growth factor stimulation, the scaffold protein, Gab1, is tyrosine phosphorylated and subsequently the adaptor protein, Crk, transmits signals from Gab1. We have previously shown that Crk overexpression, which is detectable in various human cancers, induces tyrosine phosphorylation of Gab1 without extracellular stimuli. In the present study, the underlying mechanisms were further investigated. Mutational analyses of CrkII demonstrated that the SH2 domain, but not the SH3(N) or the regulatory Y221 residue of CrkII, is critical for the induction of Gab1Y307 phosphorylation. SH2 mutation of CrkII also decreased the interaction with Gab1. In GST pull-down assay, Crk-SH2 bound to wild-type Gab1, whereas Crk-SH3(N) interacted with the Gab1 mutant, which lacks the clustered tyrosine region (residues 242-410). Tyrosine phosphorylation of Gab1 was induced by all Crk family proteins, but not other SH2-containing signalling adaptors. Src-family kinase inhibitor, PP2, abrogates Crk-induced tyrosine phosphorylations of Gab1. Y307 phosphorylation was undetectable in fibroblasts lacking Src, Yes, and Fyn, even upon overexpression of Crk, whereas cells lacking only Yes and Fyn still contained Gab1 with phosphorylated Y307. Furthermore, Crk induced the phosphorylation of Src-Y416; accordingly the interaction between Crk and Csk was increased. The Gab1-Y307F mutant failed to localize near the plasma membrane even upon HGF stimulation and decreased cell migration. Moreover, Gab1-Y307F disturbed the localization of Crk, FAK, and paxillin, which are the typical components of focal adhesions. Taken together, these results indicate that Crk facilitates tyrosine phosphorylation of Gab1-Y307 through Src, contributing to the organization of focal adhesions and enhanced cell migration, thereby possibly promoting human cancer development.

Keywords: Crk, Gab1, Src, tyrosine 307 phosphorylation

Cell Research (2009) 19:638-650. doi: 10.1038/cr.2009.40; published online 7 April 2009

\section{Introduction}

Mammalian cells possess complex signalling networks

Correspondence: Shinya Tanaka

Tel: +81-11-706-7806; Fax: +81-11-706-5902

E-mail: tanaka@med.hokudai.ac.jp

${ }^{6}$ Present address: Department of Molecular Pathobiology, Hokkaido University Research Center for Zoonosis Control, Sapporo, Japan.

Received 12 March 2008; revised 14 August 2008; accepted 15 October 2008; published online 7 April 2009 activated in response to various extracellular stimuli and tyrosine phosphorylation is a common modification for generating signals resulting in diverse cellular responses $[1,2]$. In addition to the generation of forward signals in signalling cascades, many studies have also identified positive or negative feedback signals. For instance, the negative role of Sprouty in fibroblast growth factor signalling is now well established [3].

Scaffold proteins possessing multiple protein-binding domains such as members of the p130 $0^{\text {Cas }}$ IRS, FRS, and 
Dok families are known to play important roles in tyrosine kinase-mediated signalling pathways [4]. The Gab family of scaffold proteins, including mammalian Gab1, Gab2, and Gab3, Drosophila Dos, and Caenorhabditis elegans Soc1, have been shown to associate with signalling mediators such as Grb2, SHP2, PI-3 kinase, PLC- $\gamma$, and Crk and CrkL, and are involved in transducing various signals provoked by growth factors, cytokines, or antigens $[4,5]$. Gab proteins possess multiple tyrosines, which upon phosphorylation, become binding sites for specific SH2 domains, as well as proline-rich sequences for $\mathrm{SH} 3$ interactions and a $\mathrm{PH}$ domain for the recognition of certain plasma membrane lipids. Gab proteins were shown to function downstream of EGFR, PDGFR, and FGFR family receptors, and to mediate important developmental signals of the HGF receptor c-Met [6]. It has been reported that Src is involved in HGF-dependent Gab1 tyrosine-phosphorylation [7]. Gab1 is indispensable for embryogenesis and its knockout mice die at an early embryonic stage with multiple organ defects [8].

The signalling adaptor protein Crk was originally isolated as an avian sarcoma virus CT10-encoded oncogene product, v-Crk [9]. The longer cellular protein, CrkII, is composed of one $\mathrm{SH} 2$ and two SH3 domains, whereas its alternative splicing form, CrkI, contains, similar to v-Crk, only one SH3 domain [10]. Upon appropriate extracellular stimuli, Crk proteins bind to the tyrosine-phosphorylated forms of $\mathrm{p} 130^{\text {Cas }}$, paxillin, and Cbl through their SH2 domains [11], concomitantly relocalizing the guanine-nucleotide exchange factors, Dock180 and $\mathrm{C} 3 \mathrm{G}$, through the $\mathrm{SH} 3(\mathrm{~N})$ domain [12, 13] and thereby activating the small GTPases, Rac1 and Rap1, respectively [14]. In some contexts, CrkII becomes tyrosine-phosphorylated on its Y221 residue by c-Abl. The resultant intramolecular binding of the CrkII-SH2 domain to pY221 is believed to shut down signalling [11]. Moreover, very recent structural analyses revealed that SH3 targets are dissociated from pCrkII in this context [15]. Beyond physiological cell signalling, Crk has also been reported to be indispensable for human tumorigenesis [16-18].

Upon HGF stimulation of target cells, Crk binds to phosphorylated Gab1 and then transmits the signals to small GTPases through C3G and Dock180 [19, 20]. However, we have previously found that even in the absence of growth factor stimulation, forced expression of Crk could induce a remarkable tyrosine phosphorylation of Gab1 [18]. These results suggest that Crk may generate a feedback loop signal to Gab1. In this study, we focused on the mechanism and elucidated that Crk induces tyrosine phosphorylation of Y307 in Gab1 through Src. We further show that this phosphorylation event is indispensable for the organization of focal adhesions and enhanced cell migration.

\section{Results}

Crk-SH2 domain is required for tyrosine phosphorylation of Gabl

In order to clarify the detailed mechanisms of Crkinduced tyrosine phosphorylation of Gab1, we utilized several expression plasmids for wild-type or mutant forms of Gab1 and CrkII (Figure 1A). In this context, we identified Tyr307 of Gab1 as a phospho-residue, by immunoblot analysis with antibody to phosphorylated form of Gab1-Y307 (Figure 1B; the specificity of this antibody was shown in Supplementary information, Figure S1). Y307 is part of one of the six YXXP motives in Gab1, corresponding to the Crk-SH2 domain binding consensus motif. The SH2 mutant CrkII-R38V failed to induce phosphorylation of Gab1-Y307, but CrkII-W169L, which possesses a mutation in the classical binding pocket of the N-terminal SH3 domain of CrkII, led to phosphorylated Gab1-Y307 at a slightly lower magnitude relative to that of wild-type CrkII (CrkII-WT) (Figure 1B). To exclude a potential role of tyrosine phosphorylation of CrkII itself in Gab1 phosphorylation, we utilized a CrkIIY221F mutant and confirmed that this mutation did not negatively affect Gab1-Y307 phosphorylation (Figure 1B). These data indicate that CrkII-mediated Y307 phosphorylation of Gab1 depends on the $\mathrm{SH} 2$ domain of CrkII, but not on its SH3 domain or Y221 residue.

\section{Binding of Crk to Gabl via different modes involving the SH2 or SH3(N) domain}

To determine regions responsible for Crk-Gab1 binding, we performed immunoprecipitation analyses using $293 \mathrm{~T}$ cell lysates transfected with wild-type or mutant forms of CrkII and Gab1. CrkII-WT, CrkII-W169L, and CrkII-Y221F efficiently co-precipitated wild-type Gab1 (Gab1-WT), although the Y221F mutant showed reduced binding (Figure $1 \mathrm{C}$ ). The $\mathrm{SH} 2$ mutant, CrkIIR38V, had attenuated binding to Gab1-WT (Figure 1C). When we used the Gab1 deletion mutant (Gab1del) lacking the central region (amino acids 242 to 410) of Gab1, previously designated as CBR (Crk binding region) [20], wild-type, W169L and Y221F mutant of CrkII precipitated much less Gab1-del than Gab1-WT (Figure 1C). In contrast, CrkII-R38V bound to Gab1-del better than Gab1-WT. These data suggest that, in addition to the SH2-mediated binding, the SH3(N) domain of CrkII may also interact with Gab1.

Subsequently, we performed GST precipitation assays using the CrkII-SH2 or -SH3(N) domains fused to 
A

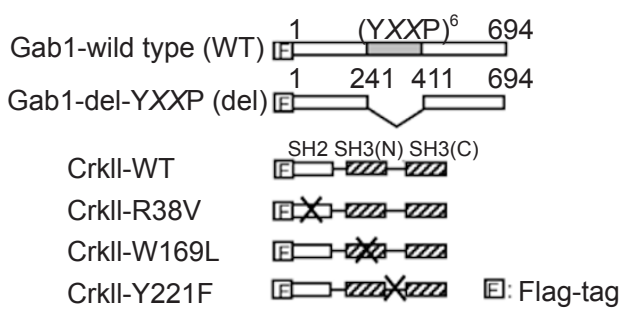

B

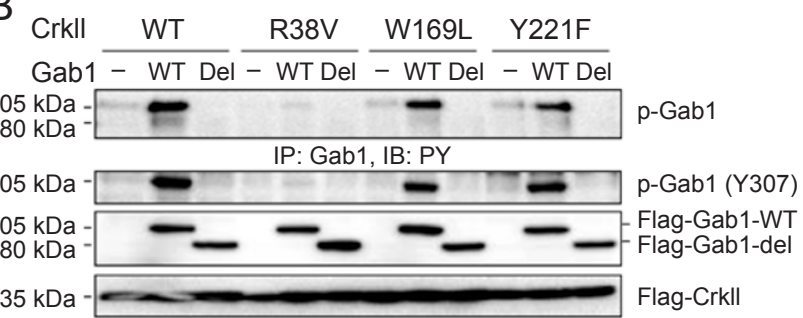

C

Crkll $\frac{\text { WT }}{\text { Gab1- WT Del }} \frac{\text { R38V }}{- \text { WT Del }} \frac{\text { W169L }}{- \text { WT Del }} \frac{\text { Y221F }}{- \text { WT Del }}$

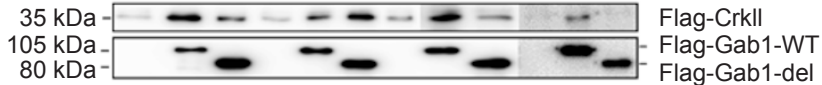

IP: Gab1, IB: Flag

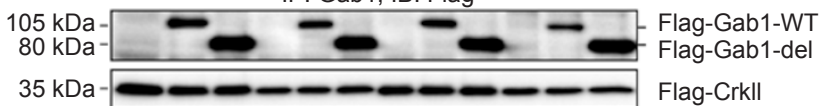

D

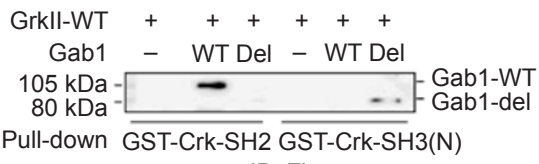

IB: Flag

$E$
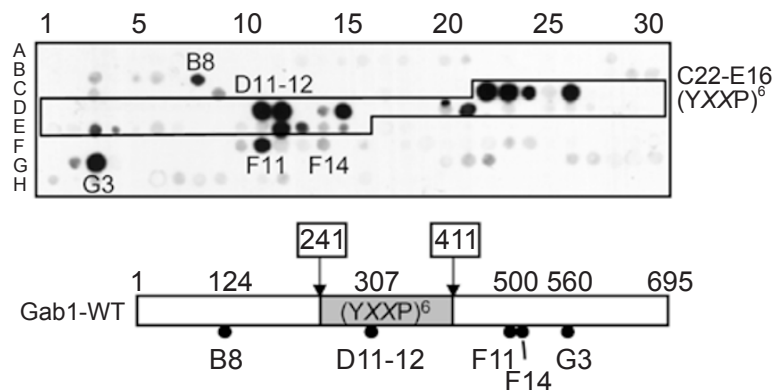

\begin{tabular}{|c|c|}
\hline B8 & CDICGFNPTEEDPVKPPGSSL \\
\hline D11 & RHVSISYDIPPTPGNTYQIPR \\
\hline D12 & SISYDIPPTPGNTY $Q I$ IRTFP \\
\hline F11 & DFSSEGMQVPPPAHMGFRSSP \\
\hline F14 & PPPAHMGFRSSPKTPPRRPVP \\
\hline G3 & APVRSPITRSFARDSSRFPMS \\
\hline
\end{tabular}

Figure 1 Crk-SH2 domain-mediated tyrosine phosphorylations of Gab1. (A) Schematic structures of Gab1, Crkll, and their mutant proteins utilized in this study. F, Flag epitope tag; WT, wild type; del, Gab1 deletion mutant lacking the six-clustered YXXP motifs. (B) Crk-SH2 domain-mediated tyrosine phosphorylation of Gab1. 293T cells were transiently co-transfected with vectors for wild-type or its mutant forms of Flag-tagged Gab1 and Flag-tagged Crkll as indicated, and their expression levels were confirmed by immunoblot analysis with antibody to Flag. Protein extracts immunoprecipitated with anti-Gab1 antibody were subjected to immunoblot analysis with antibody to phosphotyrosine (PY, top panel). Y307-specific phosphorylation of Gab1 was examined by immunoblotting using anti-phospho-Gab1 (Y307) antibody. (C) 293T cells were transiently transfected with vectors for Flag-Gab1 and Flag-Crkll as indicated. To detect the binding of Gab1 and Crkll, protein lysates immunoprecipitated with anti-Gab1 antibody were subjected to immunoblot analysis using anti-Flag antibody. (D) Flag-tagged wild-type Crkll was expressed into 293T cells with Flag-tagged wild-type Gab1 or its deletion form, and protein extracts were incubated with either GST-Crk-SH2 or GST-Crk-SH3(N) proteins. After GST-fusion precipitation, samples were subjected to immunoblot analysis with antibody to Flag. (E) Far Western overlay blot of membrane-arrayed overlapping peptides corresponding to the human Gab1 protein using GST-tagged human Crkll as a probe (top panel). The schematic structure of human Gab1 shows some key residues, and the locations of Crkll binding peptides are indicated. The sequences of the corresponding peptides are given below.

GST. CrkII and Gab1 were co-transfected into 293T cells to generate the phosphorylated form of Gab1 and cell lysates were incubated with affinity-purified GST-SH2 or GST-SH3(N) proteins. The SH3(N) domain of CrkII bound only to Gab1-del but not Gab1-WT as expected (Figure 1D). We also confirmed that the SH2 domain of CrkII bound only to Gab1-WT but not Gab1-del (Figure 1D) as reported previously [20]. Furthermore, we attempted to characterize the Crk binding region of Gab1 by peptide array analysis. Human full-length CrkII or mouse CrkII SH3(N), but not the CrkII SH3(C) domain, bound to several peptides derived from human Gab1, preferentially to the D11-12 peptides, which include the Y307 residue (Figure 1E). In this context, no binding was detected with the GST-tag alone (data not shown). In addition to the tyrosine-clustered region (corresponding to $\mathrm{C} 22$ to E16 peptides) of Gab1, CrkII also bound to $\mathrm{B} 8$ and F14 peptides containing $\mathrm{P} X X \mathrm{P}$ sequences (Figure $1 \mathrm{E})$. We also found an interaction between CrkII and the F11, which contains the entire sequences identified as the 
c-Met binding site [21] and G3 peptides of Gab1 (Figure $1 \mathrm{E})$. Taken together, these results suggest a potentially complex binding mode for Crk and Gab1.

Crk family adaptor proteins induce tyrosine phosphorylation of Gab1

To examine whether tyrosine phosphorylation of Gab1 could be induced by all members of the Crk-family of adaptors, CrkI, CrkII, CrkL or v-Crk, or other SH2containing signalling proteins (Shc, Grb2, and SHP-2) were individually overexpressed in 293T cells. By immunoprecipitation analysis, we found that all members of the Crk family could induce tyrosine phosphorylation of Gab1, whereas Shc, Grb2, and SHP-2 could not (Figure 2; their exogenous expression levels were shown in Supplementary information, Figure S2). The phosphorylation of Gab1-Y307 was also specifically observed in the presence of Crk family of adaptors (Figure 2). These data suggest that the induction of Gab1 tyrosine phosphorylation may be restricted to Crk family proteins. It is noteworthy that two different tags (Myc and Flag) on CrkII did not affect the levels of CrkII-induced Gab1 phosphorylation (Figure 2).

\section{Src family kinases phosphorylate Gabl}

As Crk does not possess catalytic activity but is known to activate tyrosine kinases of the Src family, we focused on the identification of the kinases with the potential to trigger the phosphorylation of Gab1 in 293T cells. For this analysis, several tyrosine kinases were individually overexpressed with wild-type Gab1 in 293T cells and the phosphorylation status of Gabl was examined. Expression of Src, Fyn, or Lyn resulted in tyrosine-phosphorylated Gab1, as detected by both anti-phosphotyrosine and anti-phospho Gab1-Y307 antibodies (Figure 3A). Expression of a constitutively active mutant of Src (Y527F) led to enhanced phosphorylation of Gab1 relative to that induced by wild-type Src (Figure 3A).

To confirm the involvement of Src family kinases in the phosphorylation of Gab1-Y307, we utilized the compound, PP2, a widely used inhibitor of Src family kinases [22]. PP2 markedly suppressed CrkII-induced tyrosinephosphorylation of Gab1 on Y307, while PP3, an analogue of PP2 without inhibitory activity on Src family kinases, did not affect Gab1 phosphorylation (Figure 3B).

Src kinase is indispensable for the phosphorylation of Gab1 tyrosine 307

To define more precisely which endogenously expressed Src family kinase is responsible for Crk-induced Gab1 phosphorylation, we examined the phosphostatus of Gab1 in mouse embryonic fibroblasts (MEF) derived

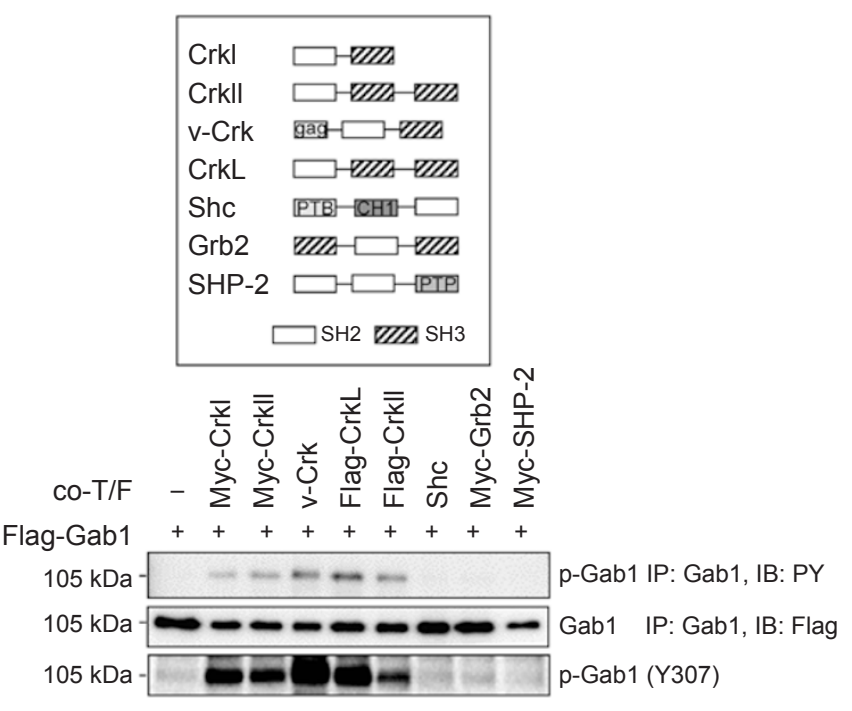

Figure 2 Crk-family-induced tyrosine phosphorylation of Gab1. Flag-tagged wild-type Gab1 was expressed into 293T cells with the individual $\mathrm{SH} 2$ domain-containing signaling proteins as indicated. Protein extracts immunoprecipitated with anti-Gab1 antibody were subjected to immunoblot analysis using antiphosphotyrosine (PY, top) or anti-Flag (middle) antibodies. Cell lysates were also directly subjected to immunoblot analysis with antibody to phosphorylated Gab1-Y307 (bottom). Schematic structures of co-transfected proteins are shown in the panel.

from animals with disrupted src, yes, fyn, and fak genes. In wild-type MEF, basal tyrosine phosphorylation of Gab1, including that on the Y307 residue, was readily detectable without the expression of exogenous CrkII and/or Gab1 (Figure 3C). Phosphorylation of Gab1Y307 was not only observed in wild-type MEF, but also, and even slightly enhanced, in MEF lacking yes and fyn $\left(\mathrm{yes}^{-/-} \mathrm{fyn}^{-/-}\right.$; designated as YF) or fak alone $\left(\mathrm{fak}^{-/-}\right.$) (Figure 3C). However, Gab1 pY307 was not detectable in MEF lacking src, yes, and fyn $\left(\mathrm{src}^{-/} \mathrm{yes}^{-/-} \mathrm{fyn}^{-/}\right.$; SYF $)$. The overall tyrosine phosphorylation of Gab1 in SYF-MEF was similar to that in wild-type MEF.

We also investigated the effects of CrkII overexpression on the phosphorylation of Gab1 in YF- or SYFMEF (Figure 3D; exposure time of blot for signal detection was shorter than that in Figure 3C). The expression of CrkII and Gab1 enhanced overall Gab1 phosphorylation, but no enhancement of Gab1 pY307 was observed in SYF (Figure 3D). The phosphorylation levels of Gab1 seem to be saturated in MEF lacking yes and fyn; therefore the potential effect of CrkII could not be evaluated (Figure 3D). These data indicate that phosphorylation of Gab1-Y307 occurs through Src kinase, while other tyrosine residues of Gab1 could be phosphorylated by other tyrosine kinases. 


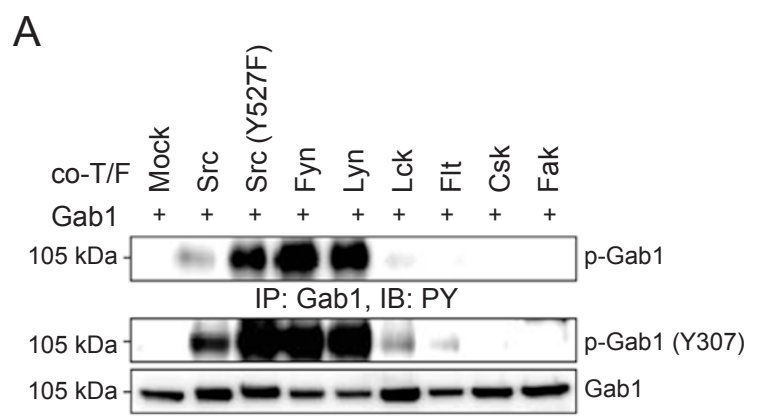

B

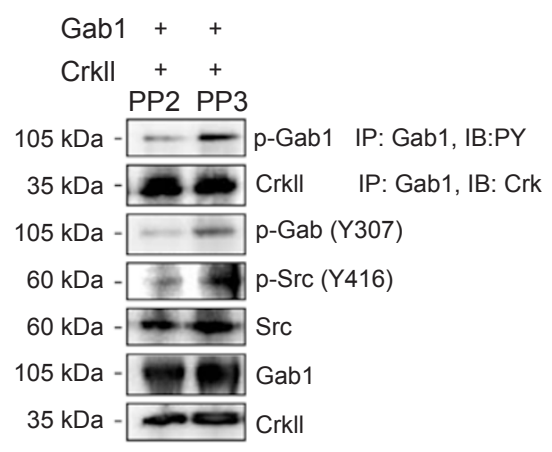

C

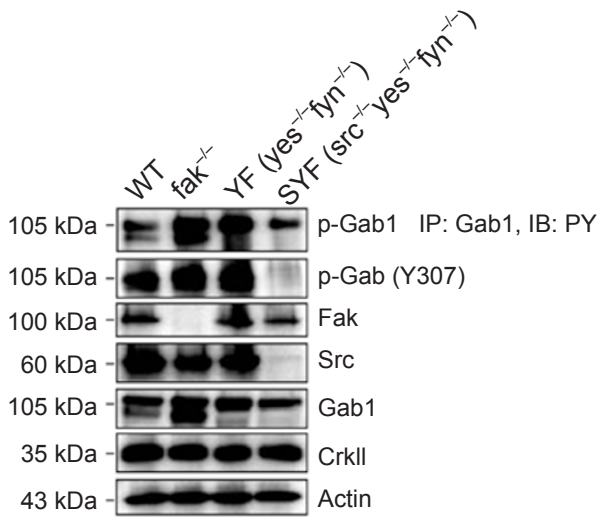

$\mathrm{D}$

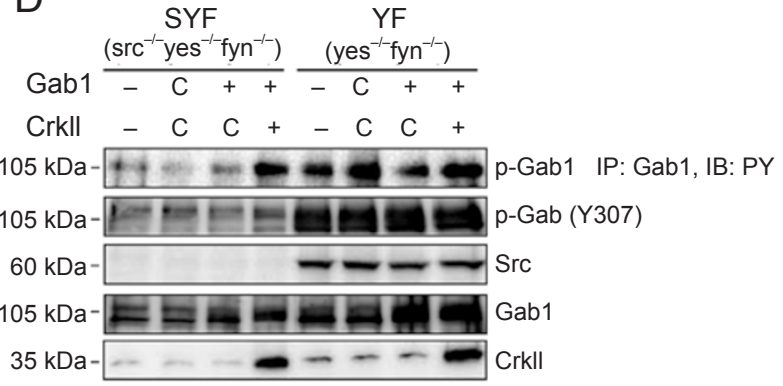

Figure 3 Src-family kinase triggers tyrosine phosphorylation of Gab1. (A) 293T cells were co-transfected with vectors for wildtype Gab1 and various tyrosine kinases as indicated. The lysate immunoprecipitated with anti-Gab1 antibody was subjected to immunoblotting using anti-phosphotyrosine antibody (PY, top). Protein extracts were also directly subjected to immunoblot analysis with antibodies to phospho-Gab1-Y307 (middle) or to Gab1 (bottom). (B) Src-family kinase inhibitor PP2 suppresses Crk-induced phosphorylations of Gab1. 293T cells expressing both Gab1 and Crkll were treated with PP2 (10 $\mu$ M) or PP3 (10 $\mu \mathrm{M})$ for $2 \mathrm{~h}$ prior to lysis and protein extracts were analyzed by immunoblotting using indicated antibodies. Cell lysates immunoprecipitated with anti-Gab1 antibody were subjected to immunoblot analysis using anti-phosphotyrosine (PY) and antiCrk antibodies. (C) Protein extracts derived from MEFs with genetic backgrounds as indicated were subjected to immunoblot analysis using the indicated antibodies. The lysates immunoprecipitated with anti-Gab1 antibody were analyzed with antiphosphotyrosine antibody (PY, top panel). (D) SYF ( $\left.\mathrm{src}^{-/-} \mathrm{yes}^{-/-} \mathrm{fyn}^{-/-}\right)$and YF $\left(\mathrm{yes}^{-/-} \mathrm{fyn}^{-/-}\right)$MEFs were transfected with vectors for Gab1 and Crkll as indicated. Protein extracts were analyzed by immunoblotting using the indicated antibodies. The lysates immunoprecipitated with anti-Gab1 antibody were subjected to immunoblot analysis with antibody to phosphotyrosine (PY, top panel). "C" indicates empty control plasmid.

Crk facilitates Src kinase activity via interaction with Csk

To determine whether increased Crk expression, which is found in some human cancers, influences the catalytic activity of Src, we examined the phosphorylation status of the regulatory tyrosine in the activation loop of the Src kinase domain, commonly designated as "Tyr416". This numbering is derived from chicken c-Src protein and corresponds to Tyr419 in human c-Src. Expression of wild-type CrkII in 293T cells clearly induced the phosphorylation of this residue as detected by immunoblotting using anti-phospho-Src Y416-specific antiserum, whereas expression of Gab1 alone did not (Figure 4A). R38V, W169L, or Y221F mutant forms of CrkII led to weaker phosphorylation of Src Y416 relative to wild- type CrkII (Figure 4B) and we also found a similar phosphorylation pattern of Src Y416 in the presence of Gab1Y307F mutant (Supplementary information, Figure S3). On the other hand, the expression of Gab1 deltaCBR substantially decreased Src pY416 (Figure 4B). As expected, in $293 \mathrm{~T}$ cells expressing various Crk-family of adaptors, pY416 levels of Src were well correlated with the phosphorylation levels of Gab1, especially on its pY307 residue (Figure 2 and Supplementary information, Figure S4).

The SH3 domain of Crk is known to bind to prolinerich sequences, especially to $\mathrm{P} X X \mathrm{P} X \mathrm{~K}$. To explore the mediator implicated in Crk-induced Src activation, we focused on Csk, a negative regulator for Src kinase by specifically phosphorylating the Y527 residue of Src, 

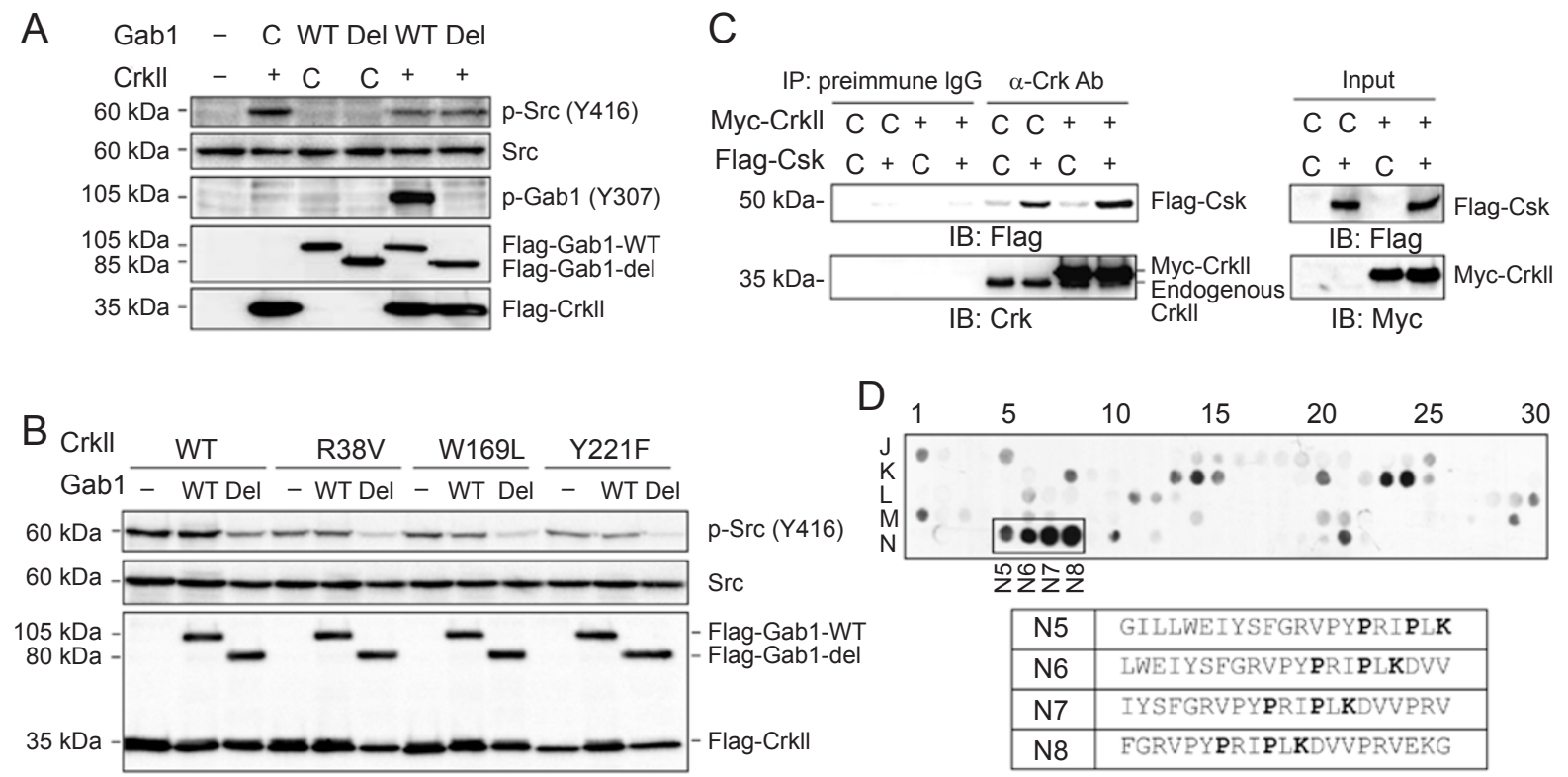

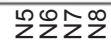

Figure 4 Crk overexpression facilitates activation loop phosphorylation of Src via interaction with Csk. (A) Flag-tagged Gab1 and Flag-tagged Crkll were co-expressed into 293T cells as indicated, and protein extracts were subjected to immunoblot analysis using the indicated antibodies. The activation status of Src was examined by immunoblotting with anti-phosphoSrc Y416-specific antibody. "C" indicates empty control plasmid. (B) 293T cells were transfected with expression vectors for wild-type or its mutant forms of Crkll and Gab1, and cell lysates were subjected to immunoblot analysis with antibodies to phosphorylated Y416 of Src, to total Src, and to Flag. (C) Association between Crk and Csk. Flag-Csk and Myc-Crkll were transiently co-expressed into 293T cells, and their expression levels were examined by immunoblotting using anti-Flag and anti-Myc antibodies, respectively. To detect the association of Csk and Crkll, protein extracts immunoprecipitated with antiCrk antibody were subjected to immunoblot analysis with antibody to Flag. Protein extracts were also precipitated with preimmune IgG as a control. "C" indicates empty control plasmid. (D) Far Western overlay blot of membrane-arrayed overlapping peptides corresponding to the human Csk protein using GST-tagged human Crkll as a probe (top panel). The sequences of prominent Crk binding peptides that share a PXXPXK motif are given below.

because Csk has a PRIPLK sequence. We found that Csk bound to Crk in $293 \mathrm{~T}$ cells by immunoprecipitation analysis (Figure 4C). Peptide array analyses further revealed that human full-length CrkII may primarily bind to the PRIPLK motif in Csk, which is localized from amino acids 388 to 393 (contained in peptides N5 to N8) (Figure 4D). These important findings clearly warrant further exploration, for example, through future analyses of relevant human tumors and matched normal control tissues for a correlation between Crk overexpression and Src pY416 levels.

Y307 residue of Gab1 is important for enhanced cell migration

To investigate the physiological role of Gab1-Y307 phosphorylation, we first examined the subcellular localization of endogenous Gab1 in MDCK cells, which have an abundance of HGF receptor c-Met. In the quiescent MDCK cells, the lined localization of Gab1 along the cell-cell contacts with condensed actin filaments, in ad- dition to the distribution in the cytoplasm, was observed (Supplementary information, Figure S5, upper panels, arrowheads). Upon HGF stimulation, a proportion of Gab1 clearly translocated to the plasma membrane, especially in regions with typical membrane ruffling (Supplementary information, Figure S5, lower panels, arrows), suggesting that Gab1 might be involved in the organization of actin cytoskeleton and its related cell movement. To examine the involvement of Gab1 pY307 in cell movement, we generated expression plasmids for GFP-fused wild-type Gab1 and its Y307F mutant, which were introduced into MDCK cells. Time-lapse analysis of the fluorescence images clearly demonstrated that upon HGF stimulation, wild-type Gab1 rapidly accumulated at the leading edges of cells, corresponding to the forefront of the movement (Figure 5A, upper panels, arrowheads), leading to the dynamic morphological changes and promoting cell migration (Supplementary information, Video S1A). In contrast, Gab1-Y307F was distributed into the cytoplasm even upon HGF stimulation, and the cells exhibited only 
A

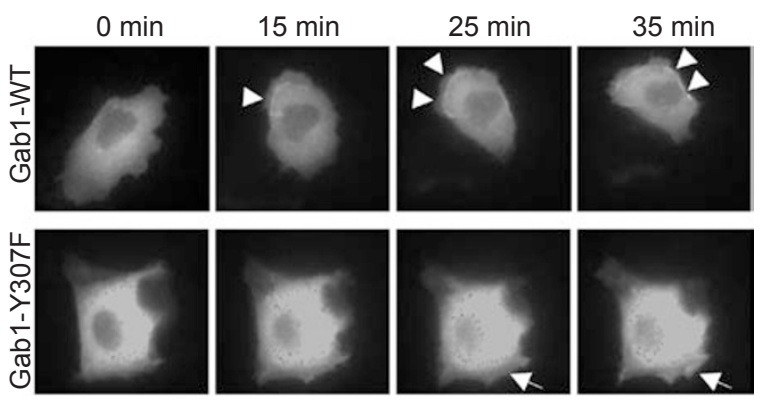

B
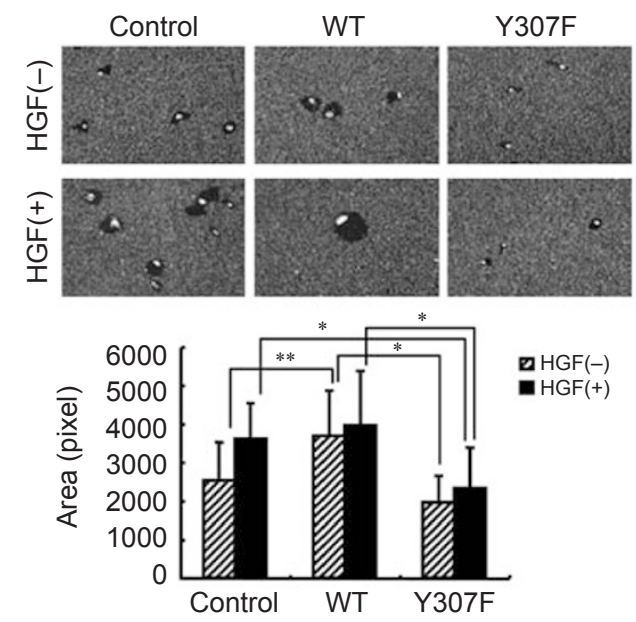

C

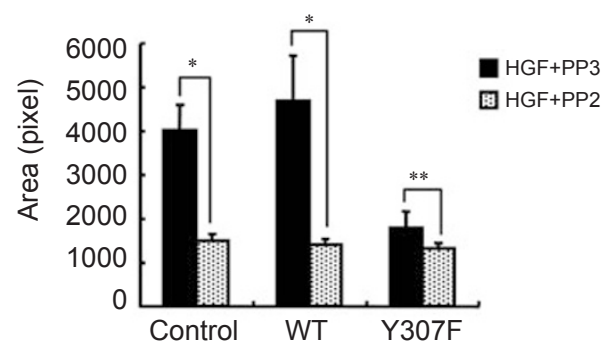

Figure 5 Tyr307 of Gab1 regulates membrane localization of Gab1 and enhances cell migration. (A) MDCK cells expressing either GFP-fused wild-type Gab1 (upper panels) or Y307F mutant (lower panels) were replaced onto glass-based dishes. Immediately after $50 \mathrm{ng} / \mathrm{ml}$ HGF stimulation, fluorescent images were obtained every 5 min with the use of a time-lapse imaging system. (B) Analysis of cell motility by phagokinetic track assay. MDCK cells stably expressing wild-type Gab1 or its Y307F mutant, or its control cells transfected with the corresponding empty vector were incubated in the absence or presence of HGF (50 $\mathrm{ng} / \mathrm{ml}$ ) for $28 \mathrm{~h}$. The motility of individual cells was determined by measuring the area cleared of gold particles. Representative micrographs are shown in the upper panels, and the quantitative data are shown in the lower panels (mean $\pm \mathrm{SD}$ ). $* P<0.0001$; $* * P<0.001$. (C) MDCK cells stably expressing wild-type Gab1 or its Y307F mutant, or its control cells were incubated in the presence of PP2 $(10 \mu \mathrm{M})$ or PP3 $(10 \mu \mathrm{M})$ with HGF treatment (50 $\mathrm{ng} / \mathrm{ml}$ ) for $28 \mathrm{~h}$, and the cell motility was analyzed as described in (B). The quantitative data are shown in the panel (mean \pm SD). $* P<0.0001 ; * * P<0.001$. a small degree of membrane extension (Figure 5A, lower panels, arrows, and Supplementary information, Video S1B).

To determine a role of Gab1 pY307 in cell motility, we established MDCK cells stably expressing wild-type Gab1 or its Y307F mutant. The endogenous expression level of Gab1 in MDCK cells was low, and we successfully obtained several cell lines stably expressing GFPfused wild-type Gab1 or its Y307F protein (Supplementary information, Figure S6). We could fortunately establish MDCK cells with an approximately equal amount of wild-type Gab1 or its Y307F protein (WT: 4 clone, Y307F: 1 clone) and confirmed similar cell growth in these cell lines (Supplementary information, Figures S6 and S7), and these cells were utilized for the analysis of cell movement. Phagokinetic track assay, which has the advantage of analyzing the random motility of a single cell, revealed that the motility of control MDCK cells was facilitated upon HGF stimulation (Figure 5B). Forced expression of wild-type Gab1 promoted cell motility, especially in the absence of HGF, whereas the Y307F mutant impaired both basal and HGF-enhanced cell motility (Figure 5B). HGF-triggered increase in cell motility in control or wild-type Gab1-expressing MDCK cells was completely inhibited by the Src family tyrosine kinase inhibitor PP2 (Figure 5C). In a wound-healing assay, we failed to observe significant alterations of cell motility between control-vector-, wild-type Gab1-, or its Y307F mutant-transfected MDCK cells in the absence of HGF, although the movement of Gab1-Y307F-expressing cells seems to be slower (Supplementary information, Figure S8A). We could also not detect the effect of HGF stimulation in the wound-healing assay (Supplementary information, Figure S8B), probably due to the insensitivity to HGF of the confluent cells to HGF.

\section{Y307 phosphorylation of Gab1 is required for the orga-} nization of focal adhesion components

Considering that the Gab1-Y307F mutant failed to accumulate at the leading edge and to enhance cell motility (Figure 5A and 5B), Gab1 pY307 might be involved in the organization of focal adhesion components including Crk, which is essential for cell motility. To clarify this, we first examined the intracellular movement of Gab1 and Crk proteins in living cells by the time-lapse imaging technique. GFP-fused wild-type Gab1 or its Y307F mutant was introduced into MDCK cells with RFP-fused CrkI. In the quiescent MDCK cells, wild-type Gab1 and CrkI were clearly co-localized to a part of the plasma membrane, appearing as a zipper-like structure (Figure 6A, upper left panel, arrowheads; unmerged images are shown in Supplementary information, Figure S9). HGF 

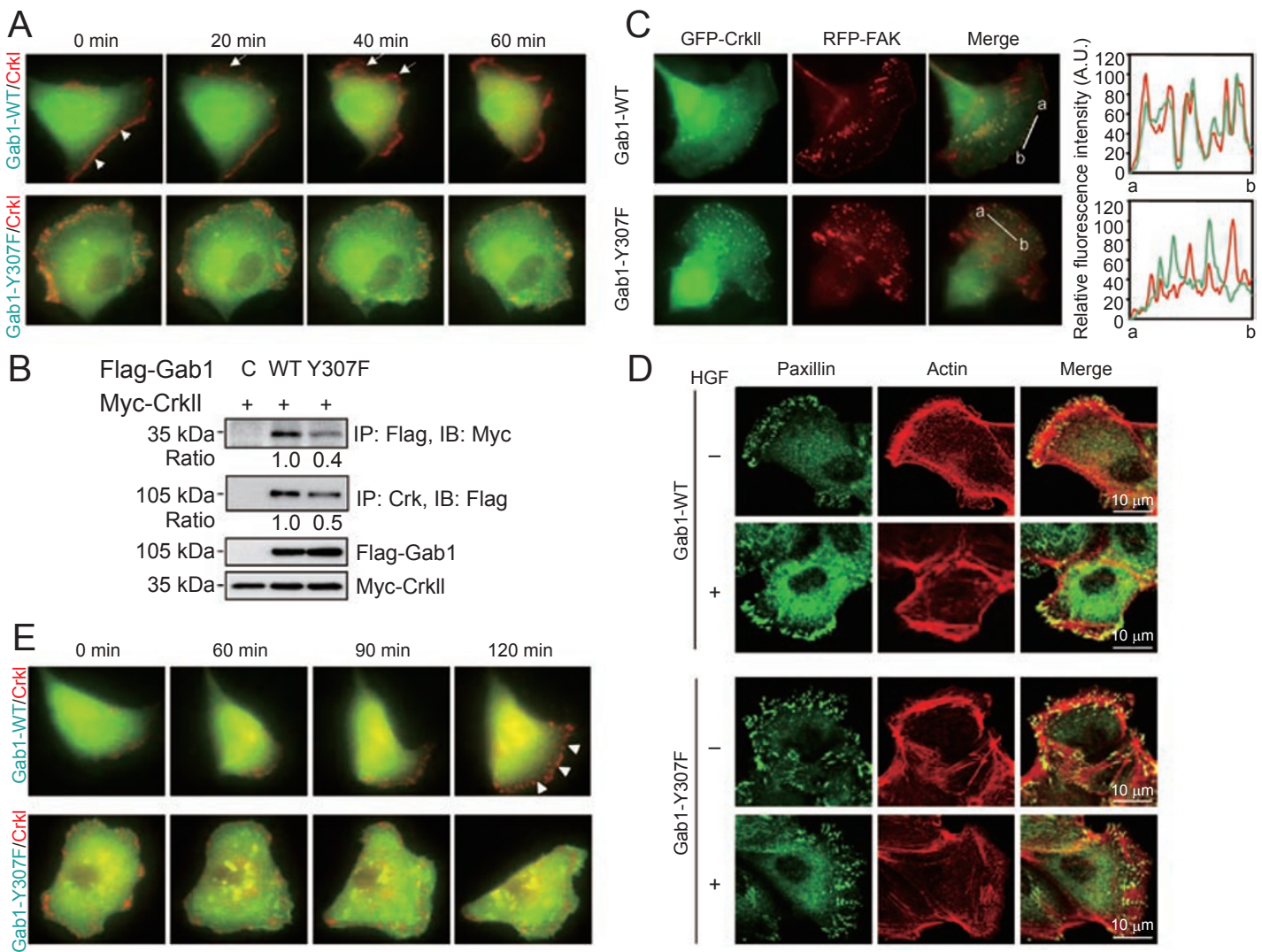

Figure 6 Phosphorylation of Gab1-Y307 residue is important for organization of focal adhesion components. (A) RFP-fused Crkl were overexpressed in MDCK cells with either GFP-fused wild-type Gab1 or its Y307F mutant. Immediately after 50 ng/ ml HGF stimulation, fluorescent images were obtained every 1 min using a time-lapse system. Green color indicates Gab1 protein, whereas Red exhibits Crkl. (B) 293T cells were transfected with expression vectors for Flag-Gab1 WT or Flag-Gab1 Y307F, or with the corresponding empty vector $\mathrm{C}$ in the presence of Myc-Crkll, and cell lysates were subjected to immunoprecipitation with antibodies to Flag or to Crk, and the resulting precipitates were subjected to immunoblot analysis with antibodies to Myc or to Flag, respectively. Cell lysates were also directly subjected to immunoblot analysis with antibodies to Flag or to Myc. (C) MDCK cells stably expressing Gab1-WT or its Y307F mutant were transfected with expression vectors for GFP-fused Crkll and RFP-fused FAK, and the fluorescent images were acquired. Fluorescence intensities of GFP (green) and RFP (red) along with the line (from a to b) in merged images were analyzed using MetaMorph software, and are plotted in the right panels. Note that the overlapped peaks indicate co-localization of GFP-Crkll and RFP-FAK. (D) MDCK cells stably expressing Gab1-WT or its Y307F mutant were incubated in the absence or presence of HGF (50 ng/ml) for $30 \mathrm{~min}$, and cells were subjected to immunofluorescence analysis with antibody to paxillin and with phalloidin. Merged images are shown in the right panels. (E) MDCK cells expressing GFP-fused wild-type Gab1 or its Y307F mutant with RFP-fused Crkl were preincubated with PP2 $(10 \mu \mathrm{M})$ for $2 \mathrm{~h}$. Immediately after $50 \mathrm{ng} / \mathrm{ml}$ HGF stimulation, fluorescent images were obtained as described in (A).

stimulation led to some cytoplasmic protrusions, and CrkI was rapidly translocated to the periphery (Figure 6A, upper panels, arrows). In contrast, in MDCK cells with Gab1-Y307F expression, CrkI was detected as disordered spots at the inside of the plasma membrane, and even upon HGF stimulation, CrkI could not move to the edge of the plasma membrane (Figure 6A, lower panels). Supporting these results, immunoprecipitation analysis revealed that the single mutation of Gab1-Y307F diminished the binding to Crk to less than half relative to that of wild-type Gab1 (Figure 6B). These results suggest that Gab1 pY307 might have a role in recruiting Crk to the leading edge of cells in response to growth factor stimulation, possibly through the binding of Crk to the Gab1 pY307 residue.

We further examined whether Gab1-Y307 is also involved in the organization of other focal adhesion components. In wild-type Gab1-expressing MDCK cells, we observed the clear co-localization of CrkII and FAK, a representative component of focal adhesion, especially 
along the plasma membrane with ruffles (Figure 6C, upper panels). Paxillin, another typical component of focal adhesion, was also lined around the plasma membrane in these cells in an orderly manner, and it obviously accumulated to the membrane upon HGF stimulation (Figure $6 \mathrm{D}$, upper six panels). On the other hand, MDCK cells with Gab1-Y307F expression displayed disorganized localization of Crk and FAK (Figure 6C, lower panels), and paxillin (Figure 6D, lower six panels), and Crk did not co-localize with FAK (Figure 6C, lower panels). Taken together, these results suggest that Gab1 pY307 is important for the proper organization of focal adhesion components.

Finally, to determine the implication of Src family kinases and c-Met kinase in Gab1 phosphorylation, MDCK cells were pre-incubated with PP2 followed by HGF stimulation. In wild-type Gab1-expressing cells, the zipper-like structures involving Crk and Gabl disappeared upon PP2 pre-treatment (compare upper left panels of Figure 6A and 6E). HGF stimulation of the cells led to membrane extensions, and CrkI clearly accumulated to the cell periphery (compare Figure 6A and 6E, upper panels, arrowheads). In contrast, the cells with Gab1Y307F expression exhibited the disturbed localization of Crk all the time, resulting in disordered cell morphology (Figure 6E, lower panels). All of the above-mentioned time-lapse images are shown in Supplementary information, Video S2.

\section{Discussion}

Tyrosine kinase-mediated signal transduction is involved in numerous cellular functions including cell growth, motility, and differentiation in response to various external stimuli. Upon HGF stimulation of cells, the HGF receptor c-Met tyrosine-phosphorylates Gab1, allowing Crk and CrkL to bind to several epitopes in Gab1, which leads to the transmission of signals for cell growth, motility, or epithelial-mesenchymal transition, depending on the affected cell type [4, 23] (Figure 7A).

In this study, we show that enhanced expression of Crk increases the presence of tyrosine-phosphorylated Gab1 through Src family tyrosine kinases. In particular, we found that phosphorylation of the Gab1-Y307 residue depends on Src (Figure 7Bii). Y307 is embedded in the motif YDIP, which conforms to the general Crk-SH2 domain-binding consensus sequence, $\mathrm{YXX \textrm {P }}$, and binding of Crk to this tyrosine may be a part of the positive feedback loop mediated by Src. Gab1 is also known to receive a feedback signal from the S/T-kinase Erk2, which phosphorylates Gab1 on other residues [24]. Since many phosphorylation sites exist in Gab1, feedback loop
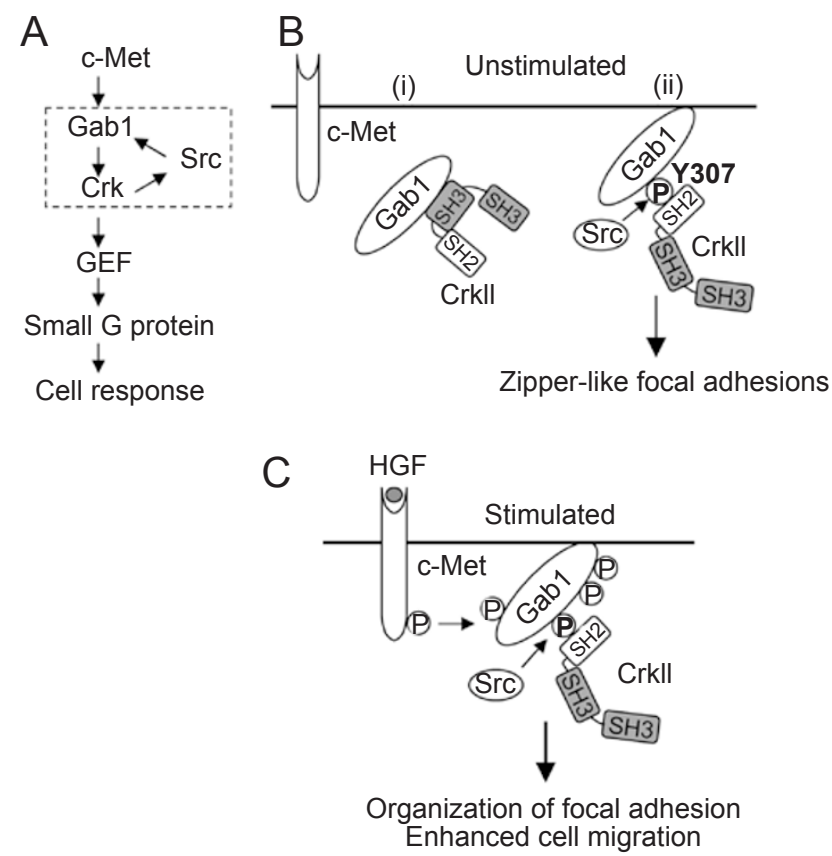

Figure 7 Schematic diagram of Crk-mediated signaling related to Gab1. (A) Crk-mediated feedback signal to Gab1. Crkinduced Gab1 phosphorylation through Src activation may function as a feedback signal (dotted box), thereby adding to wellestablished forward signaling from the receptor tyrosine kinase through the signaling cascade $\mathrm{Met} \rightarrow \mathrm{Gab} 1 \rightarrow \mathrm{Crk} \rightarrow \mathrm{GEF} \rightarrow$ small G protein. GEF, guanine nucleotide exchange factor. (B, C) Working model for interaction modes between Crk and Gab1. (B) In quiescent cells, Crk may continuously bind to Gab1 via different modes depending on the $\mathrm{SH} 2$ or $\mathrm{SH} 3(\mathrm{~N})$ domain. (i) Crk appears to possess the ability to bind to the motifs except for the clustered tyrosines of Gab1 through its $\mathrm{SH} 3(\mathrm{~N})$ domain. (ii) Enhanced Crk expression facilitates Src kinase activity, leading to Tyr307 phosphorylation of Gab1, followed by the binding of Crk through its $\mathrm{SH} 2$ domain. (C) Upon stimulation of cells by growth factors, Gab1 is multiple tyrosine-phosphorylated and Crk rapidly switches the binding mode to Gab1 through its preferential $\mathrm{SH} 2$ domain.

signals through Erk2, Crk/Src and possibly other kinases may be a common theme in Gab1 signalling. Gab1 may therefore be an important signal integration point for numerous kinase signals. In addition, PLC $-\gamma$ is also known to bind to Y307 of Gab1 and Crk may therefore modulate PLC- $\gamma$-dependent signal transduction [25].

In the context of this study, we also attempted to investigate the physiological role of Gab1-Y307 tyrosine phosphorylation. Therefore, we generated an expression plasmid for the Gab1-Y307F mutant and examined potential cellular phenotypes upon the co-expression of Crk and Gab1-Y307F. Considering the results that mutation of the Y307 residue of Gab1 disturbed the proper local- 
ization of Crk, FAK, and paxillin and suppressed cell migration, the binding of Crk to the Y307 residue may be critical for the organization of focal adhesions regulating cell motility. As the Y307 residue was also identified as a potential SH2-independent binding site for Crk by peptide array analysis, each of the six Crk binding motives that reside in Gab1, may individually mediate the specific cellular phenotypes. For example, PLC- $\gamma$ has been shown to bind to the Gab1 tyrosines 373 and 407 [25]; so these tyrosine residues contribute to other cellular phenotypes. Considering that overexpression of $\mathrm{Crk}$ has been observed in various human cancers [17] and that in sarcoma cells Crk plays a role in HGF signalling [18], the continuous phosphorylation of Gab1 through a positive feedback mechanism involving Crk might contribute to the malignant transformation of tumor cells, for example, by promoting cell migration.

We also unexpectedly found that Crk can bind to Gab1 through its SH3(N) domain. This raises the interesting possibility that CrkII can also bind to motifs other than the clustered tyrosine region in Gab1 in non-stimulated cells (Figure 7Bi). Peptide array analysis implicated one PXXP motif of Gab1 as a potential SH3 domainbinding site (Figure 1E). Upon tyrosine phosphorylation of Gab1, Crk may undergo an interaction switch now binding to Gab1 via the SH2 domain (Figure 7C) and transmitting signals through kinases and/or GEF proteins interacting with the Crk SH3(N) domain. This interaction mode should render Crk available for rapidly transmitting signals from Gabl.

Of several known Gab1 interacting proteins that were tested, only Crk family adaptors were found to increase the tyrosine phosphorylations of Gab1. This result suggested to us that Crk might contribute to the tyrosine phosphorylation of Gab1, not only by its protection of Gab1 phosphoepitopes from phosphatases, but also by another mechanism. To follow up on this, we examined the involvement of tyrosine kinases in Crk-induced Gab1 phosphorylation and found that Crk enhances tyrosine phosphorylation of Gab1 through Src.

The mechanism of Src activation through Crk has not been clarified, although Crk was previously reported to activate Src in the context of v-Crk-induced transformation of chicken embryonal fibroblasts [26]. In the present study, we showed that overexpression of Crk alone is sufficient to facilitate the phosphorylation on the regulatory Y416 residue of Src (Figure 4A), which indicates an increase in the catalytic activity of the Src kinase domain. To investigate the underlying mechanism, we focused on Csk, a key regulatory kinase for the Src family kinases. Csk is known to phosphorylate the Y527 residue of Src, which results in the negative regulation of Src kinase activity $[27,28]$. In co-transfection studies, we found co-precipitation of Crk with Csk (Figure 4C). Furthermore, peptide array analysis revealed that Crk has an ability to bind to the $\mathrm{P} X X \mathrm{P} X \mathrm{~K}$-containing epitope in Csk, which is not entirely unexpected (Figure 4D) and indicates a potential direct interaction between Crk and Csk. These observations highlight the intriguing possibility that Crk may prevent Csk from interacting with Src, likely resulting in the activation of Src and subsequent phosphorylation of Gab1. Further in-depth analyses of this point are needed.

Our current studies also lead us to conclude that the Src-interacting kinase, Fak, is unlikely to be prominently involved in Crk-dependent phosphorylation of Gab1, since Fak-deficient MEF are fully able to phosphorylate Gab1 (Figure 3C). Furthermore, the kinase activity of Fak, estimated by the phosphorylation status of Fak, was not affected by the overexpression of CrkII in 293T cells (Supplementary information, Figure S10).

In summary, our data demonstrate that a Crk-dependent feedback signal can induce tyrosine phosphorylation of Gab1-Y307, in the absence of extracellular stimuli, through the activation of Src, which is critical for an appropriate organization of focal adhesions and enhanced cell migration. Since Crk is overexpressed in several human cancers, the feedback loop signal demonstrated here may act to promote the malignant phenotypes of cancers; therefore, prevention of Crk-induced Gab1 phosphorylation through Src inhibition might be a therapeutic option for some cancers.

\section{Materials and Methods}

\section{Cell culture}

Mouse embryonic fibroblasts (MEF) were prepared from postnatal day 13 embryos. SYF $\left(\mathrm{src}^{-/-}\right.$yes $\left.{ }^{-/} \mathrm{fyn}^{-/-}\right)$and $\mathrm{S}++\left(\mathrm{yes}^{-/-}\right.$ $f \mathrm{yn}^{-/-}$) (in this manuscript designated YF hereafter) MEF were purchased from ATCC (Manassas, VA, USA) and fak-deficient MEF [29] were a kind gift from M. Hamaguchi (Nagoya University, Japan). All cells used in this study, including $293 \mathrm{~T}$ human embryonic kidney cells and MDCK (NBL-2) dog kidney cells (JCRB9029), were maintained in Dulbecco's modified Eagle's medium supplemented with $10 \%$ fetal bovine serum and incubated at $37^{\circ} \mathrm{C}$ in a humidified atmosphere containing $5 \% \mathrm{CO}_{2}$. For treatment with an Src family inhibitor, PP2, or its control compound, PP3 $(10 \mu \mathrm{M}$; Calbiochem, San Diego, CA, USA) were added to culture media and the cells were incubated for $48 \mathrm{~h}$.

To address the physiological role of Gab1 pY307, MDCK cells were stably transfected with expression plasmids for EGFPfused wild-type Gab1 or EGFP-fused Gab1-Y307F, or with the corresponding empty vector. The exogenous expression of Gab1 was examined by immunoblot analysis with antibody to Gab1, and MDCK cells with an approximately equal amount of wild-type Gab1 or its Y307F protein (WT: 4 clone, Y307F: 1 clone) were utilized for the analysis. 


\section{Expression plasmids and transfection}

Wild-type and mutant (R38V, W169L, Y221F) human CrkII cDNAs as well as cDNAs for human CrkL and chicken Csk were subcloned into the pCXN2-Flag expression vector [30]. Fulllength cDNA for human CrkI and CrkII as well as retroviral v-Crk were subcloned into the pCAGGS-Myc [30] and/or pMex [10] expression vectors. Mouse Gab1 cDNAs for wild-type and Y307F mutant were subcloned into $\mathrm{pBat}$-Flag and $\mathrm{pEGFP}$ expression vectors (Clontech, Palo Alto, CA, USA). Other expression plasmids were obtained from the following sources: pBat-Flag-Gab1-deltaYXXP [20]; pCAGGS-RFP-CrkI, pEGFP-CrkII, pCAGGS-RFPFAK (from N. Mochizuki, National Cardiovascular Cent. Res. Inst., Japan); pKU-hyg-Shc [31] (from N. Gotoh, University of Tokyo, Japan); pSP65SRa-Grb2-Myc and pSP65SRa-SHP2-Myc [32] (from H. Higashi, Hokkaido Univ., Japan); pCA-Fyn, pMexLyn, pMik-cSrc, pMik-cSrc-Y527F, pMex-Lck, pMex-Flt-1 and pcDNA-Csk (from H. Hanafusa, Osaka Bioscience Institute, Osaka, Japan); pRcCMV-FAK-HA-WT (from S. K. Hanks, Vanderbilt Univ., USA). Transfections were carried out using Fugene 6 or HD (Roche, Indianapolis, IN, USA) according to the manufacturer's directions.

\section{GST precipitation assay for analysis}

Protein extracts derived from 293 T cells expressing CrkII and Gab1 (either wild-type or delta CBR mutant) were incubated with the GST fusion proteins, GST-Crk-SH2, or GST-Crk-SH3(N), as described previously [33]. Samples were then subjected to immunoblot analysis.

\section{Immunoprecipitation and immunoblotting}

Cell lysis, protein determination, immunoblotting and immunoprecipitation were carried out as described previously [34]. Antibodies were obtained from the following sources: anti-phosphotyrosine $(\mathrm{RC} 20 \mathrm{H})$, anti-Crk, and anti-Fak from BD Transduction Laboratories (Lexington, KY, USA); anti-Gab1 and anti-Src from Upstate (Lake Placid, NY, USA); anti-phospho Gab1 (pY307) and anti-phospho Src (pY416) from Cell Signaling (Beverly, MA, USA); anti-Flag (M2) from Sigma (Saint Louis, MO, USA); antiMyc from Invitrogen (Carlsbad, CA, USA); anti-pan-actin from Chemicon (Temecula, CA, USA).

\section{Peptide array overlay blots of Csk and Gabl sequences for detection of potential Crk binding sites}

Peptide arrays on cellulose filters were synthesized on a Multipep Synthesiser (Intavis Bioanalytical Instruments AG, Cologne, Germany) and kindly provided by the CR-UK Peptide Synthesis Group (Nicola O'Reilly, LRI, London, UK). Human Csk and Gab1 were generated as arrays of overlapping peptides of 21 amino acid lengths each, covering the full protein length. Following the first peptide, corresponding to the N-terminus of the protein, each subsequent peptide generated was shifted by 3 amino acids. Peptides are assembled, one amino acid at a time, by sequential coupling and deprotection, on a derivatized cellulose membrane (aminoPeg500 UC540; Intavis). After synthesis, the dried membrane was briefly pre-wetted in ethanol, then washed with TBST $(20 \mathrm{mM}$ Tris- $\mathrm{HCl}(\mathrm{pH} 7.5), 100 \mathrm{mM} \mathrm{NaCl}, 0.1 \%$ [v/v] Tween 20) 3 times for $10 \mathrm{~min}$ and blocked in TBST with $5 \%[\mathrm{w} / \mathrm{v}]$ non-fat dry milk for $4 \mathrm{~h}$ at room temperature. The membrane was then incubated with $8 \mu \mathrm{g} / \mathrm{ml}$ of affinity chromatography-purified GST in a block- ing buffer overnight at $4{ }^{\circ} \mathrm{C}$ to detect background signals elicited by the GST-tag. After washing 3 times in TBST for $10 \mathrm{~min}$, it was probed with an anti-GST mAb, washed as before with TBST and incubated with HRP-coupled secondary (anti-Ig) antibody. Detection of bound GST was attempted by ECL, but no signals were observed even after prolonged exposure ( 2 hours). The membrane was then washed again in TBST, reblocked as detailed before and reprobed with $20 \mu \mathrm{g} / \mathrm{ml}$ GST-CrkII (human, full length; DNA kindly provided by M Matsuda) overnight at $4{ }^{\circ} \mathrm{C}$. Bound GSTCrk fusion protein was detected as described above for GST and the signals were apparent after a few seconds.

\section{Immunofluorescence analysis}

MDCK cells stably expressing either wild-type Gab1 or its Y307F mutant were incubated in the absence or presence of HGF (50 ng/ml) for $30 \mathrm{~min}$, and fixed with 3\% paraformaldehyde for 15 min and then permeabilized with $0.1 \%$ Triton X-100 in PBS for $4 \mathrm{~min}$ at room temperature. Cells were incubated with $1 \%$ bovine serum albumin to block non-specific binding of antibodies. The cells were incubated with anti-paxillin antibody (BD Transduction Laboratories), and subsequently with Alexa Fluor 488-conjugated secondary antibody (Molecular Probes). To visualize actin filaments, the cells were also stained with Alexa Fluor 594-conjugated phalloidin (Molecular Probes). Fluorescent images were obtained using a confocal laser-scanning microscope (FV-300; Olympus, Tokyo, Japan).

\section{Time-lapse-imaging}

MDCK cells expressing either GFP-fused wild-type Gab1 or its Y307F mutant, in some contexts also containing RFP-fused CrkI, were seeded onto glass-based dishes. Immediately after $50 \mathrm{ng} / \mathrm{ml}$ HGF stimulation, fluorescent images were obtained every $1 \mathrm{~min}$ using a time-lapse microscopic system (Nippon Roper, Chiba, Japan). Images were analyzed using MetaMorph software (Molecular Devices, Downingtown, PA, USA). When examining the involvement of Src family kinases, cells were preincubated with $10 \mu \mathrm{M}$ PP2 for $2 h$.

\section{Phagokinetic track assay}

Random cell motility was assessed by the phagokinetic track assay developed by Albrecht-Buehler [35]. Briefly, carpets of gold particles were prepared on cover glasses $(18 \times 18 \mathrm{~mm}$; Matsunami, Japan) coated with $1 \%$ bovine serum albumin. The gold particlecoated cover glasses were placed in $35-\mathrm{mm}$ tissue culture dishes, and 3000 MDCK cells stably transfected with vectors for wildtype Gab1 or Gab1-Y307F, or with the corresponding control vector, were plated on the cover glasses and incubated in the absence or presence of HGF $(50 \mathrm{ng} / \mathrm{ml})$. When examining the involvement of Src family kinases, cells were also incubated in the presence of PP2 $(10 \mu \mathrm{M})$ or its control analogue PP3 $(10 \mu \mathrm{M})$. After $28-\mathrm{h}$ incubation, the cover glasses were fixed in $10 \%$ formalin for 30 min and mounted on slide glasses. The phagokinetic tracks were observed with a microscope at a magnification of $\times 200$, and the area cleared of gold particles by randomly selected 30 cells on each condition was measured using MetaMorph software (Molecular Devices). All data represent means and standard deviations from a single experiment, and were subject to two-way analysis of variance, followed by comparison by Student's $t$-test. $P$ values obtained from the test are described in the figure legends. 


\section{Visualization of focal adhesions}

MDCK cells stably expressing wild-type Gab1 or its Y307F mutant were co-transfected with the expression vectors for GFPfused CrkII and RFP-fused FAK. After $24 \mathrm{~h}$ of transfection, the cells were replated onto glass-based dishes and further incubated for $2 \mathrm{~h}$. Fluorescent images were acquired using a fluorescent microscope (Nippon Roper).

\section{Acknowledgments}

We thank M Hamaguchi (Nagoya Univ., Japan) and T Iwahara (Osaka Bioscience Institute, Japan) for fak null MEFs, and N Gotoh (Tokyo Univ., Japan), H Higashi (Hokkaido Univ., Japan), N Mochizuki (National Cardiovascular Cent. Res. Inst., Japan), H Hanafusa (Prof. emeritus, The Rockefeller Univ., USA; and Director em., OBI, Japan), SK Hanks (Vanderbilt Univ., USA), and M Matsuda (Kyoto Univ., Japan) for plasmids. We also thank K Sasai (Hokkaido Univ., Japan) and Y Ohba (Hokkaido Univ., Japan) for valuable discussion. This work was supported in part by grants-inaid from the Ministry of Education, Science, Culture, and Sports, and the Ministry of Health, Labor, and Welfare, Japan, as well as Suhara Memorial Foundation (Sapporo, Japan), and the Mochida Medical Science Foundation (Tokyo, Japan). The work of SF and TK is supported by grants from Cancer Research UK and the British Cancer Charity "Heads Up".

We dedicate this work to our great mentor Hidesaburo Hanafusa, professor emeritus of the Rockefeller University, who passed away on March 15, 2009 at the age of 79. He devoted his life to science and in particular to creating the oncogene research field, to teaching and to providing profound affection to his students and postdocs. All of the alumni of Saburo's laboratory pride themselves in having been his apprentices and we would like to hereby express our deeply felt gratitude to Saburo.

\section{References}

1 Schlessinger J. Cell signaling by receptor tyrosine kinases. Cell 2000; 103:211-225.

2 Yaffe MB. Phosphotyrosine-binding domains in signal transduction. Nat Rev Mol Cell Biol 2002; 3:177-186.

3 Hanafusa H, Torii S, Yasunaga T, Nishida E. Sproutyl and Sprouty2 provide a control mechanism for the Ras/MAPK signalling pathway. Nat Cell Biol 2002; 4:850-858.

$4 \mathrm{Gu}$ H, Neel BG. The "Gab" in signal transduction. Trends Cell Biol 2003; 13:122-130.

5 Nishida K, Hirano T. The role of Gab family scaffolding adapter proteins in the signal transduction of cytokine and growth factor receptors. Cancer Sci 2003; 94:1029-1033.

6 Furge KA, Zhang YW, Vande Woude GF. Met receptor tyrosine kinase: enhanced signaling through adapter proteins. Oncogene 2000; 19:5582-5589.

7 Chan PC, Chen YL, Cheng CH, et al. Src phosphorylates Grb2-associated binder 1 upon hepatocyte growth factor stimulation. J Biol Chem2003; 278:44075-44082.

8 Schaeper U, Vogel R, Chmielowiec J, Huelsken J, Rosario M, Birchmeier W. Distinct requirements for Gab1 in Met and EGF receptor signaling in vivo. Proc Natl Acad Sci USA 2007; 104:15376-15381.
9 Mayer BJ, Hamaguchi M, Hanafusa H. A novel viral oncogene with structural similarity to phospholipase C. Nature 1988; 332:272-275.

10 Matsuda M, Tanaka S, Nagata S, Kojima A, Kurata T, Shibuya M. Two species of human CRK cDNA encode proteins with distinct biological activities. Mol Cell Biol 1992; 12:34823489 .

11 Feller SM, Knudsen B, Hanafusa H. c-Abl kinase regulates the protein binding activity of c-Crk. EMBO J 1994; 13:23412351 .

12 Hasegawa H, Kiyokawa E, Tanaka S, et al. DOCK180, a major CRK-binding protein, alters cell morphology upon translocation to the cell membrane. Mol Cell Biol 1996; 16:1770-1776.

13 Tanaka S, Morishita T, Hashimoto Y, et al. C3G, a guanine nucleotide-releasing protein expressed ubiquitously, binds to the Src homology 3 domains of CRK and GRB2/ASH proteins. Proc Natl Acad Sci USA 1994; 91:3443-3447.

14 Feller SM. Crk family adaptors-signalling complex formation and biological roles. Oncogene 2001; 20:6348-6371.

15 Kobashigawa Y, Sakai M, Naito M, et al. Structural basis for the transforming activity of human cancer-related signaling adaptor protein CRK. Nat Struct Mol Biol 2007; 14:503-510.

16 Linghu H, Tsuda M, Makino Y, et al. Involvement of adaptor protein Crk in malignant feature of human ovarian cancer cell line MCAS. Oncogene 2006; 25:3547-3556.

17 Nishihara H, Tanaka S, Tsuda M, et al. Molecular and immunohistochemical analysis of signaling adaptor protein $\mathrm{Crk}$ in human cancers. Cancer Lett 2002; 180:55-61.

18 Watanabe T, Tsuda M, Makino Y, et al. Adaptor molecule Crk is required for sustained phosphorylation of Grb2-associated binder 1 and hepatocyte growth factor-induced cell motility of human synovial sarcoma cell lines. Mol Cancer Res 2006; 4:499-510

19 Lamorte L, Royal I, Naujokas M, Park M. Crk adapter proteins promote an epithelial-mesenchymal-like transition and are required for HGF-mediated cell spreading and breakdown of epithelial adherens junctions. Mol Biol Cell 2002; 13:14491461.

20 Sakkab D, Lewitzky M, Posern G, et al. Signaling of hepatocyte growth factor/scatter factor (HGF) to the small GTPase Rap1 via the large docking protein Gab1 and the adapter protein CRKL. J Biol Chem 2000; 275:10772-10778.

21 Schaeper U, Gehring NH, Fuchs KP, Sachs M, Kempkes B, Birchmeier W. Coupling of Gab1 to c-Met, Grb2, and Shp2 mediates biological responses. J Cell Biol 2000; 149:14191432.

22 Hanke JH, Gardner JP, Dow RL, et al. Discovery of a novel, potent, and Src family-selective tyrosine kinase inhibitor. Study of Lck- and FynT-dependent T cell activation. J Biol Chem 1996; 271:695-701.

23 Boccaccio C, Comoglio PM. Invasive growth: a MET-driven genetic programme for cancer and stem cells. Nat Rev Cancer 2006; 6:637-645.

24 Roshan B, Kjelsberg C, Spokes K, Eldred A, Crovello CS, Cantley LG. Activated ERK2 interacts with and phosphorylates the docking protein GAB1. J Biol Chem 1999; 274:36362-36368

25 Gual P, Giordano S, Williams TA, Rocchi S, Van Obberghen E, Comoglio PM. Sustained recruitment of phospholipase C- 
gamma to Gab1 is required for HGF-induced branching tubulogenesis. Gene 2000; 19:1509-1518.

26 Sabe H, Shoelson SE, Hanafusa H. Possible v-Crk-induced transformation through activation of Src kinases. J Biol Chem 1995; 270:31219-31224.

27 Okada M, Nada S, Yamanashi Y, Yamamoto T, Nakagawa H. CSK: a protein-tyrosine kinase involved in regulation of src family kinases. J Biol Chem 1991; 266:24249-24252.

28 Sabe H, Okada M, Nakagawa H, Hanafusa H. Activation of cSrc in cells bearing v-Crk and its suppression by Csk. Mol Cell Biol 1992; 12:4706-4713.

29 Ilic D, Furuta Y, Kanazawa S, et al. Reduced cell motility and enhanced focal adhesion contact formation in cells from FAKdeficient mice. Nature 1995; 377:539-544.

30 Niwa H, Yamamura K, Miyazaki J. Efficient selection for high-expression transfectants with a novel eukaryotic vector.
Gene 1991; 108:193-199.

31 Gotoh N, Toyoda M, Shibuya M. Tyrosine phosphorylation sites at amino acids 239 and 240 of Shc are involved in epidermal growth factor-induced mitogenic signaling that is distinct from Ras/mitogen-activated protein kinase activation. Mol Cell Biol 1997; 17:1824-1831.

32 Higashi H, Tsutsumi R, Muto S, et al. SHP-2 tyrosine phosphatase as an intracellular target of Helicobacter pylori CagA protein. Science 2002; 295:683-686.

33 Tanaka S, Hattori S, Kurata T, et al. Both the SH2 and SH3 domains of human CRK protein are required for neuronal differentiation of PC12 cells. Mol Cell Biol 1993; 13:4409-4415.

34 Makino Y, Tsuda M, Ichihara S, et al. Elmo1 inhibits ubiquitylation of Dock180. J Cell Sci 2006; 119:923-932.

35 Albrecht-Buehler G. The phagokinetic tracks of 3T3 cells. Cell 1977; 11:395-404.

(Supplementary information is linked to the online version of the paper on the Cell Research website.) 